\title{
Emergency airway management by non-anaesthesia house officers - a comparison of three strategies
}

\author{
V Dörges, H Ocker, V Wenzel, C Sauer, P Schmucker
}

\begin{abstract}
Objectives-The purpose of this study was to determine effects of different airway devices and tidal volumes on lung ventilation and gastric inflation in an unprotected airway.

Methods-Thirty one non-anaesthesia house officers volunteered for the study, and ventilated a bench model simulating an unintubated respiratory arrest patient with bag-valve-facemask, laryngeal mask airway, and combitube using paediatric and adult self inflating bags.

Results-The paediatric versus adult self inflating bag resulted with the laryngeal mask airway and combitube in significantly $(p<0.001)$ lower mean (SEM) lung tidal volumes (376 (30) v 653 (47) $\mathrm{ml}$, and $368(28) v 727(53) \mathrm{ml}$, respectively). Gastric inflation was zero with the combitube; and 0 (0) $v 8$ (3) $\mathrm{ml}$ with the laryngeal mask airway with low versus large tidal volumes. The paediatric versus adult self inflating bag with the bag-valve-facemask resulted in comparable lung tidal volumes (245 (19) v 271 (33) $\mathrm{ml}$; $\mathrm{p}=\mathrm{NS}$ ); but significantly $(\mathrm{p}<0.001)$ lower gastric tidal volume $(149$ (11) $v 272(24) \mathrm{ml}$ ).

Conclusions-The paediatric self inflating bag may be an option to reduce the risk of gastric inflation when using the laryngeal mask airway, and especially, the bagvalve-facemask. Both the laryngeal mask airway and combitube proved to be valid alternatives for the bag-valve-facemask in this experimental model.

(Emerg Med f 2001;18:90-94)
\end{abstract}

Keywords: bag-valve-mask; laryngeal mask airway; combitube; tidal volume; gastric regurgitation

Inhospital cardiopulmonary resuscitation (CPR) response teams may consist of a non-anaesthesiologist because of shortage in experienced anaesthesiologists, or intensive care unit physicians. Also, it is quite common that hospital buildings are spread over a large area or in many different floors, or both. As such, many hospitals may not be able to dispatch experienced anaesthesiologists to remote locations for prolonged periods of time to administer CPR, as this would render a critical care unit or even operation room patients unattended. Thus, the physician in charge of an inhospital CPR attempt may be one of the clinically lesser experienced physicians, but has to carry full responsibility with regard to pharmacological interventions, and especially, airway management. Accordingly, if an airway device can be identified that is easiest to handle for this target group, CPR efforts may benefit.

For rescuers who do not have adequate skills in tracheal intubation, the most common means of providing emergency ventilatory support in non-intubated patients during CPR is the bag-valve-facemask system. ${ }^{1}$ As bag-valvefacemask ventilation has a number of disadvantages, such as leakage around the facemask, and gastric inflation, ${ }^{2-6}$ the laryngeal mask airway and the combitube may be an alternative to bag-valve-mask ventilation. ${ }^{7-14}$

Hence, the purpose of this study was to assess lung ventilation and gastric inflation when non-anaesthesia house officers perform ventilation with the bag-valve-facemask system, the laryngeal mask airway, and the combitube in a bench model. Furthermore, it was investigated whether smaller tidal volumes, as recommended by the European Resuscitation Council, ${ }^{15}$ but not by the American Heart Association, at the time of this investigation ${ }^{16}$ is beneficial in order to reduce the risk of gastric inflation as suggested by some previous bench models simulating a cardiac arrest patient. ${ }^{17-19}$

\section{Methods}

EXPERIMENTAL MODEL

A bench model simulating an unintubated cardiac arrest patient was designed with a new intubation manikin head (Bill I, VBM Medizintechnik, Sulz, Germany), and a lung simulator (LS 800, Dräger, Lübeck, Germany). Lung compliance $\left(50 \mathrm{ml} / \mathrm{cm} \mathrm{H}_{2} \mathrm{O}\right),{ }^{20-22}$ airway resistance $\left(16 \mathrm{~cm} \mathrm{H} \mathrm{H}_{2} \mathrm{O} / \mathrm{l} /\right.$ second), ${ }^{50}$ and lower oesophageal sphincter pressure $\left(6 \mathrm{~cm} \mathrm{H}_{2} \mathrm{O}\right)^{23}$ were adjusted to simulate respiratory mechanics of a cardiac arrest patient. Respiratory parameters were recorded using the AS 3 compact monitor (Datex Ohmeda, Helsinki, Finland). Subsequently, this setup was connected to a paediatric pneumotachometer to record oesophageal peak pressure, and gastric inflation (fig 1).

\section{EXPERIMENTAL PROTOCOL}

Thirty one non-anaesthesia house officers with no previous experience in emergency airway 


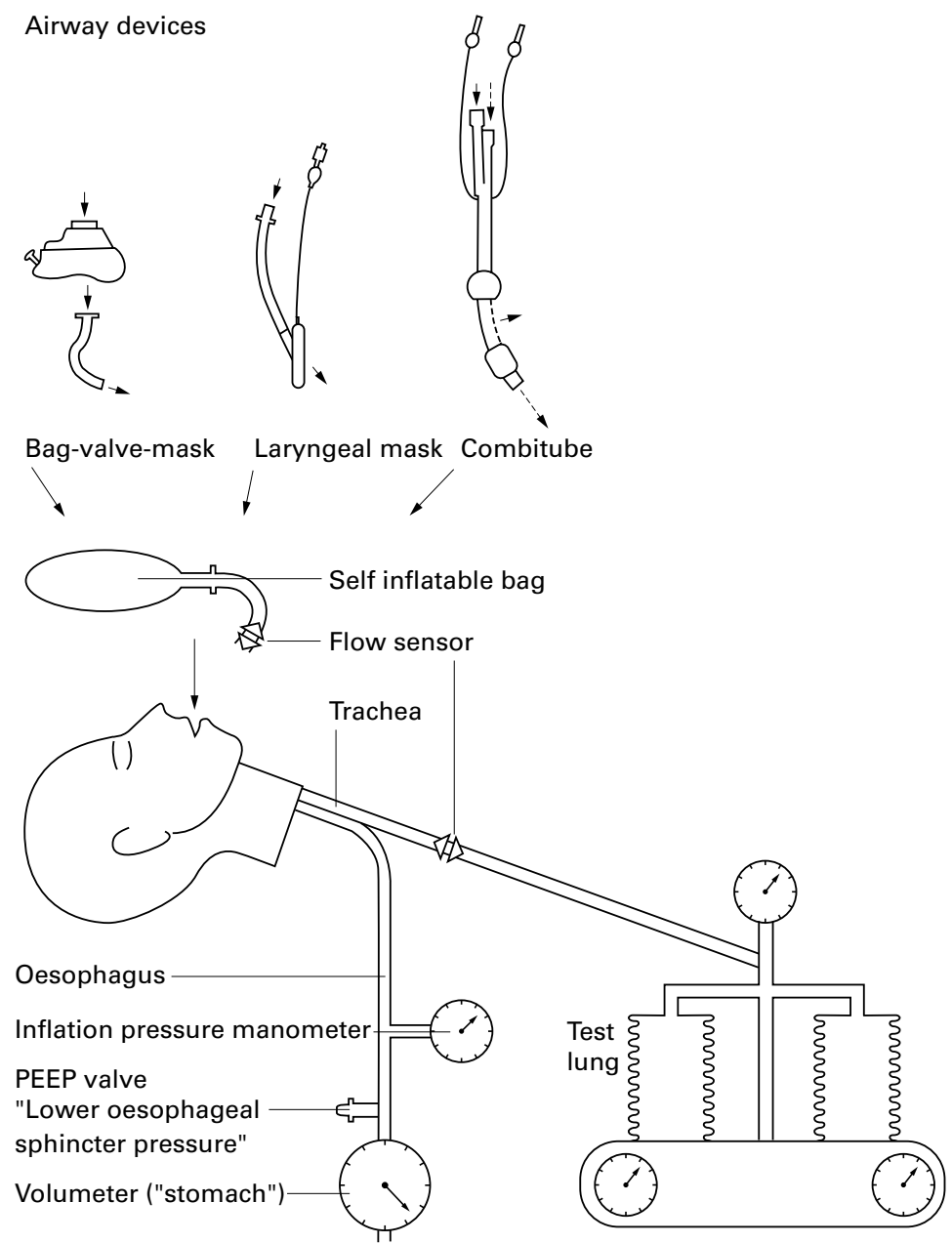

Figure 1 Modification of a previously described bench model of positive pressure ventilation with an unprotected airway. ${ }^{16}$ The upper airway was provided by a new intubation manikin head. The tracheal outlet of the manikin head was connected to a mechanical test lung (lung compliance, $50 \mathrm{ml} / \mathrm{cm} \mathrm{H} \mathrm{H}_{2} \mathrm{O}$; airway resistance, $16 \mathrm{~cm} \mathrm{H}_{2} \mathrm{O} / \mathrm{ll} / \mathrm{s}$ ). The oesophageal outlet of the manikin head was connected to an adjustable positive end expiratory pressure (PEEP) valve, which represented lower oesophageal sphincter pressure. $A$ second outlet from the PEEP valve was connected to a paediatric pneumotachometer to record oesophageal peak pressure and gastric inflation. A flow sensor was inserted between the self inflating bag and the investigated airway device; another flow sensor was inserted into the simulated trachea. The flow sensors were connected to respiratory monitors to measure ventilation variables.

management volunteered in this study; and signed written informed consent before participation.

All volunteers were instructed with theoretical and hands on teaching to appropriately use the facemask size 4 (Ambu, Hanau, Germany), a size 4 laryngeal mask airway (LMA International Services Ltd, UK), and a size 41 combitube (Tyco Sheridan, Argyle, NY) by the instructor before the study. Positioning of the combitube was confirmed using the tube check (oesophageal detector; Ambu, Hanau, Germany). For ventilation, we chose an adult and paediatric self inflating bag (maximum volume,

Table 1 Number of ventilation failures and number of volunteers inflating the stomach with each airway device

\begin{tabular}{llll}
\hline & Bag-valve-facemask & Laryngeal mask airway & Combitube \\
\hline Ventilation failures & $2 / 31$ & $3 / 31$ & $2 / 31$ \\
Stomach inflation & $29 / 29^{\star}$ & $7 / 28+$ & $0 / 29$ \\
\hline
\end{tabular}

Data are given in absolute numbers. ${ }^{\star} \mathrm{p}<0.01$ versus laryngeal mask airway and combitube. $t \mathrm{p}<0.01$ versus combitube.
1500 and $700 \mathrm{ml}$, respectively; Dräger, Lübeck, Germany). The participants then used each ventilatory device with both self inflating bags in a randomised sequence for a two minute ventilation attempt of the model simulating a cardiac arrest patient.

The time to attain the end expiratory tidal lung volume exceeding $200 \mathrm{ml}$ was recorded. If this volume could not be achieved within 180 seconds, it was deemed that the ventilation attempt had failed. Peak airway pressures at the pharynx level and in the oesophagus were recorded, as well as lung and gastric tidal volumes during each attempt. Each volunteer was graded by the instructor from 1 (excellent) to 4 (bad) in regards of airway insertion success, quality of the seal, and visible adequacy of ventilation. Accordingly, a subjective grading by the volunteer (1-4) about the different devices in conjunction with the two sizes of self inflating bags was made after the trial.

\section{STATISTICAL METHODS}

The Mann-Whitney U test was chosen for the comparison of the two self inflating bags. The Friedman test was performed to determine significant differences within the ventilatory devices. Comparison by pairs of the ventilatory devices was performed with the Wilcoxon test. Comparison in regards of stomach inflation was assessed with Fisher's exact test; $\alpha$ was set at 0.05 .

\section{Results}

Thirty one non-anaesthesia house officers (15 male, 16 female, age 29-39; specialty: two neurologists, two obstetricians/gynaecologists, two psychiatrists, six surgeons, 14 internists, three paediatricians, two ear, nose and throat surgeons, respectively) volunteered. Two participants needed $>180$ seconds to deliver a lung tidal volume of $\geqslant 200 \mathrm{ml}$ with the bag-valvefacemask, three in the laryngeal mask airway group, and two in the combitube group (table 1). The time to deliver the first adequate tidal volume ranged from 4-107 seconds (median, 14 seconds) for the bag-valve-facemask, 17129 seconds (median, 29 seconds) for the laryngeal mask airway, and 32-180 seconds (median, 61 seconds) for the combitube, respectively $(\mathrm{p}<0.01$ bag-valve-facemask $v$ laryngeal mask airway, combitube, respectively; $\mathrm{p}<0.01$ laryngeal mask airway $v$ combitube).

When the participants used the paediatric self inflating bag, tidal lung volumes with both laryngeal mask airway and combitube were significantly $(p<0.001)$ lower when compared with the adult self inflating bag. Tidal lung volume showed no significant difference between both bags in the bag-valve-facemask group. In contrast, the use of the paediatric compared with adult self inflating bag resulted in a significantly $(p<0.001)$ lower oesophageal tidal volume in the bag-valve-facemask group (table 2).

Use of the combitube resulted in a significantly $(p<0.001)$ higher tidal lung volume, and peak airway pressure with the adult self inflating bag compared with the bag-valvefacemask; furthermore, the combitube was the only ventilatory device without gastric 
Table 2 Tidal lung and tidal oesophageal volume, airway and oesophageal peak pressure for all ventilatory devices and both self inflating bags

\begin{tabular}{|c|c|c|c|c|}
\hline & $\begin{array}{l}\text { Peak } P_{a x v} \\
\left(\mathrm{~cm} \mathrm{H} \mathrm{H}_{2} \mathrm{O}\right)\end{array}$ & $\begin{array}{l}\text { Peak } P_{\text {oesoph }} \\
\left(\mathrm{cm} \mathrm{H} \mathrm{H}_{2} \mathrm{O}\right)\end{array}$ & $V_{T}$ Lung $(\mathrm{ml})$ & $V_{T}$ Oesoph $(\mathrm{ml})$ \\
\hline \multicolumn{5}{|c|}{ Bag-valve-facemask } \\
\hline Paediatric bag & $12(1)$ & $11(1)$ & $245(19)$ & $149(11)$ \\
\hline Adult bag & $14(1)$ & $12(1)$ & 271 (33) & $272(24)+$ \\
\hline \multicolumn{5}{|c|}{ Laryngeal mask airway } \\
\hline Paediatric bag & $16(1)^{\star}$ & $1(0.5)^{\star}$ & $368(28)^{\star}$ & $0(0)^{\star}$ \\
\hline Adult bag & $20(1)^{\star}$ & $3(0.5)^{\star}$ & $727(53) \dagger^{\star}$ & $8(3)^{\star}$ \\
\hline \multicolumn{5}{|l|}{ Combitube } \\
\hline Paediatric bag & $20(1)^{\star} \ddagger$ & $0^{\star}$ & $376(30)^{\star}$ & $0^{\star}$ \\
\hline Adult bag & $24(1)^{\star} \ddagger$ & $0^{\star}$ & $653(47)^{\star} \neq \dagger$ & $0^{\star}$ \\
\hline
\end{tabular}

Data are given as mean $(\mathrm{SEM}) . \mathrm{V}_{\mathrm{T}}=$ tidal volume, $\mathrm{P}=$ airway pressure, $\mathrm{P}$ = oesophageal pressure. $t \mathrm{p}<0.01$ adult bag versus paediatric self inflating bag, ${ }^{\star} \mathrm{p}<0.01$ versus bag-valvefacemask, $\neq \mathrm{p}<0.01$ versus laryngeal mask airway.

inflation. Lung and gastric volumes as well as peak airway and oesophageal pressures showed a significant $(\mathrm{p}<0.001)$ difference between the laryngeal mask airway and the bag-valvefacemask. Statistical analysis of the paediatric self inflating bag performance showed the same significant differences as the adult self inflating bag (table 2).

Both laryngeal mask airway and combitube were graded significantly $(\mathrm{p}<0.001)$ better than the bag-valve-facemask by the participants in their subjective assessment. In terms of success of airway insertion, quality of seal, and adequacy of ventilation, the instructors ranked the laryngeal mask airway and combitube significantly $(\mathrm{p}<0.01)$ better than the bag-valve-facemask as well.

\section{Discussion}

When paramedics in the Houston, Texas, Emergency Medical Service were trained to administer advanced airway management, ventilation associated complications were more related to training efforts than to the airway devices themselves. ${ }^{24}$ When extrapolating this experience to non-anaesthesia house officers in order to provide inhospital advanced cardiac life support, two pragmatic solutions may be possible: (1) extensive, continuous training; and (2) standing orders favouring an airway device that is simple to handle with little training. While paramedics, emergency physicians, and anaesthesiologists perform basic and advanced cardiac life support on a daily basis, non-anaesthesia house officers may spend a large portion of their residency in rotations without the need to handle respiratory or cardiac emergencies, or both, on a daily basis, or possibly, even never in their training. Also, training and maintaining advanced airway skills in all or at least some residents may be a cost issue, especially as money allocated to healthcare is decreasing.

The dilemma of tracheal intubation is that at least in some cases, adverse effects such as an anterior larynx, presence of vomitus in the pharynx, obesity, a short neck, or simply no adequate training may render intubation conditions extremely difficult. While an anaesthesiologist may be able to master all of these problems because of experience of several hundred or even thousands of intubations, non-anaesthesia house officers may fail to secure the airway because of lack of education or continuous training, or both. ${ }^{25}$ This has fundamental practi- cal implication. While services such as helicopter emergency medical service programmes or accident and emergency departments are always staffed with persons with an extensive and continuous background in emergency airway management, inhospital CPR response teams often consist of house officers or even nurse ${ }^{26}{ }^{27}$; while some small hospitals may not even have a CPR response team at all. For example, our volunteers reflect a wide variety of physicians from neurology, obstetrics/gynaecology, psychiatry, surgery, internal medicine, paediatrics, ear, nose and throat with no or very limited experience in emergency airway management; who participated in a class to study management of emergency patients with diseases such as myocardial infarction, stroke, respiratory, and cardiac arrest. Although none of the house officers in our group was routinely assigned to inhospital cardiac arrest teams as part of their clinical training, they had to carry full responsibility in regards of pharmacological interventions, and especially, emergency airway management during CPR. Accordingly, the chance that a non-anaesthesia physician provides emergency airway management is actually quite possible. These physicians may be a psychiatrist managing a patient with an overdose, an ENT surgeon seeing a patient with severe pharyngeal haemorrhage, an eye physician confronted with an anaphylactic shock, or an orthopaedic surgeon trying to rescue a patient with pulmonary embolus. In that case, if the response time of the cardiac arrest team is anywhere between two and five minutes, initial airway management in that time will have decided whether the stomach is inflated, or if the patient remains hypoxic or hypercarbic, or both, or if the patient is adequately ventilated and oxygenated. ${ }^{28} 29$

This study suggests that use of the laryngeal mask airway and the combitube was beneficial. The bag-valve-facemask resulted in the most gastric inflation, and insufficient pulmonary tidal volumes. However, small instead of large tidal volumes had a relative advantage, especially when using a bag-valve-facemask. Although the combitube was the only device that safely prevented gastric inflation in our bench model, the associated training commitment with this device possibly may not be guaranteed in non-anaesthesia house officers. ${ }^{25}$ Also, the time required for insertion of the combitube was significantly longer than with both bag-valvefacemask and laryngeal mask airway resulting in approximately 30 seconds longer potential hypoxaemia and hypercarbia. Therefore, for physicians with little experience in airway management, our data may tip the scales towards using the laryngeal mask airway because ease of handling, and speed of insertion. ${ }^{10} 1317$

One approach to achieve proper ventilation may be the choice of the best ventilatory device; another strategy may be a smaller tidal volume, as recently recommended by the European Resuscitation Council in order to minimise gastric inflation during ventilation of an unprotected airway. ${ }^{15} 30$ Thus, the best combination of the right "hardware", and the right tidal volume may contribute to sufficient oxygenation and ventilation, and may avoid 
gastric inflation. For example, our bag-valvefacemask group clearly demonstrates that small tidal volumes almost cut gastric inflation in half, while maintaining lung volumes. However, when using either ventilation bag with the bag-valve-facemask, only about $50 \%$ to $60 \%$ of the recommended lung tidal volumes were achieved. Both the laryngeal mask airway and especially, the combitube were the superior devices in regards of minimising or even avoiding gastric inflation with either ventilation bag. ${ }^{12}{ }^{31}$ However, it has to be pointed out that when using small tidal volumes with either ventilatory device in our bench model, lung volumes between about $250 \mathrm{ml}$ to $380 \mathrm{ml}$ were achieved, which is significantly less than the $500 \mathrm{ml}$ recommended by the European Resuscitation Council. ${ }^{15}$ This is especially important as either approximately $50 \%$ oxygen has to be used when administering tidal lung volumes of $\leqslant 400 \mathrm{ml}$, or larger tidal volumes of $\geqslant 600 \mathrm{ml}$ when room air has to be used to maintain both sufficient oxygenation and ventilation. ${ }^{32-34}$ The new international guidelines of both the ERC and AHA now recommend small tidal volumes of about 500 $\mathrm{ml}$ with an $\mathrm{FIO}_{2}$ of $>0.4$ when ventilating an unprotected airway. These recommendations are based, in part, on our experience, and further indicate the clinical significance of our observation. ${ }^{3636}$

Some limitations of our study should be noted. Firstly, our model is a tough challenge with a lower oesophageal sphincter pressure of $6 \mathrm{~cm} \mathrm{H}^{2} \mathrm{O}$, reflecting a cardiac arrest patient. ${ }^{23} 37$ Thus, the performance of our volunteers most probably would have been better when a respiratory arrest patient without cardiac arrest and subsequently, with a lower oesophageal sphincter pressure of about 20 $\mathrm{cm} \mathrm{H}_{2} \mathrm{O}$ would have been simulated. Furthermore, it is impossible to simulate changing respiratory system compliance such as during CPR, which was shown in a laboratory model. Also, we are unable to report inflation pressures, and at what pressure and tidal volume a leak occurred with each device. Although our manikin is unable to simulate the upper airway of a human perfectly in regards of sealing conditions, we suggest that peak airway pressure values in both the laryngeal mask airway and combitube group indicated an adequate seal.

In conclusion, the paediatric self inflating bag may be an option to reduce the risk of gastric inflation when using the laryngeal mask airway, and especially, the bag-valve-facemask. Both the laryngeal mask airway and combitube proved to be valid alternatives for the bag-valve-facemask in our experimental model.

Contributors

All coauthors designed the study, and collected the data. Volker Dörges and Volker Wenzel edited the first draft of the manuscript, all other coauthors subsequently made critical suggestions to the article. The final draft was read and approved by all coauthors. Volker Dörges will act as guarantor.

Presented, in part, as an abstract at the APICE Annual Meet-
Pest

Presented, in part, as an abstract at the APICE Annual Meet-
ing, Trieste, Italy, November 1998, and at the Annual Meeting ing, Trieste, Italy, November 1998, and at the Annual Meeting
of the American Society of Anesthesiologists, Dallas, Texas, of the American
October 1999.
Funding: this project was supported, in part, by the Department of Anaesthesiology, the Medical University of Lübeck, Germany.

Conflicts of interest: none.

1 The 1998 European Resuscitation Council guidelines for adult advanced life support. A statement from the Working Group on advanced life support. Resuscitation 1998;37:8190.

2 Lawes EG, Baskett PJ. Pulmonary aspiration during unsuccessful cardiopulmonary resuscitation. Intensive Care Med 1987;13:379-82.

3 Krischer JP, Fine EG, Davis JH, et al. Complications of cardiac resuscitation. Chest 1987;92:287-91.

4 Ruben H, Knudsen EJ, Carugati G. Gastric insufflation in relation to airway pressure. Acta Anaesth Scand 1961;5: relation

5 Weiler N, Heinrichs W, Dick W. Assessment of pulmonary mechanics and gastric inflation pressure during mask ventilation. Prehospital Disaster Medicine 1995;10:101-5.

6 Wenzel V, Idris AH, Lindner KH. Ventilation with an unprotected airway during cardiac arrest. In: Vincent JL, ed. Yearbook of intensive care and emergency medicine. Berlin: Springer-Verlag, 1997:483-92.

7 Doerges V, Sauer C, Ocker H, et al. Airway management during cardiopulmonary resuscitation. A comparison of bag-valve-mask, laryngeal mask and combitube. Resuscitation 1999;41:63-9.

8 Dörges V, Sauer C, Ocker H, et al. Smaller tidal volumes during cardiopulmonary resuscitation: Comparison of adult and paediatric self-inflatable bags with three different ventilatory devices. Resuscitation 1999;43:31-7.

9 Frass M, Frenzer R, Rauscha F, et al. Evaluation of the esophageal tracheal combitube in cardiopulmonary resuscitaphageal tracheal combitube in cardiop
tion. Crit Care Med 1987;15:609-11.

10 Ho-Tai LM, Devitt JH, Noel AG, et al. Gas leak and gastric insufflation during controlled ventilation: face mask versu laryngeal mask airway. Can f Anaesth 1998;45:206-11.

11 Martin PD, Cyna AM, Hunter WAH, et al. Training nursing staff in airway management: A clinical comparison of the face mask and the laryngeal mask. Anaesthesia 1993;48:33-7.

12 Frass M, Frenzer R, Zdrahal F, et al. The esophageal tracheal combitube: preliminary results with a new airway for CPR. Ann Emerg Med 1987;16:768-72.

13 Anonymous. The use of the laryngeal mask airway by nurses during cardiopulmonary resuscitation. Results of a multicentre trial. Anaesthesia 1994;49:3-7.

14 Stone BJ, Chantler PJ, Baskett PJF. The incidence of regurgitation during cardiopulmonary resuscitation: a comparigitation during cardiopulmonary resuscitation: a compari-
son between the bag valve mask and laryngeal mask airway. son between the bag valve
Resuscitation 1998;38:3-6.

15 Airway and Ventilation Management Working Group of the European Resuscitation Council. Guidelines for the basic management of the airway and ventilation during CPR. Resuscitation 1996;31:187-200.

16 Emergency Cardiac Care Committee and Subcommittees, American Heart Association: Guideline for cardiopulmonary resuscitation and emergency cardiac care. Part II: Adult basic life support. $\mathcal{F} A M A$ 1992;268:2184-8.

17 Wenzel V, Idris AH, Banner MJ, et al. Influence of tidal volume on the distribution of gas between the lungs and the stomach in the nonintubated patient receiving positive pressure ventilation. Crit Care Med 1998;26:364-8.

18 Wenzel V, Idris AH, Banner MJ, et al. Respiratory system compliance decreases after cardiopulmonary resuscitation and stomach inflation: impact of large and small tidal volumes on calculated peak airway pressure. Resuscitation 1998;38:113-18.

19 Idris AH, Wenzel V, BannerMJ, et al. Smaller tidal volumes minimize gastric inflation during CPR with an unprotected airway. [Abstract]. Circulation 1995;92:I-759.

20 Ornato JP, Bryson BL, Donovan PJ, et al. Measurement of ventilation during cardiopulmonary resuscitation. Crit Care Med 1983;11:79-82.

21 Davies K Jr, Johannigman JA, Johnson RC Jr, et al. Lung compliance following cardiac arrest. Acad Emerg Med 1995;10:855-6.

22 Safar P, Agusto-Escarraga L. Compliance in anesthetized adults. Anesthesiology 1959;20:283-9.

23 Bowman FP, Menegazzi JJ, Check BD, et al. Lower esophageal sphincter pressure during prolonged cardiac arrest and geal sphincter pressure during prolonged cardiac
resuscitation. Ann Emerg Med 1995; 26:215-19.

24 Pepe PE, Zachariah BS, Chandra NC. Invasive airway techniques in resuscitation. Ann Emerg Med 1993;22:393403.

25 Atherton GL, Johnson JC. Ability of paramedics to use the combitube in prehospital cardiac arrest. Ann Emerg Med 1993;22:1263-8.

26 Verghese C, Prior-Willeard PF, Baskett PJ. Immediate management of the airway during cardiopulmonary resuscitation in a hospital without a resident anaesthesiologist. Eur $\mathcal{F}$ Emerg Med 1994;3:123-5.

27 Tolley PM, Watts AD, Hickman JA. Comparison of the use of the laryngeal mask and face mask by inexperienced personnel. Br f Anaesth 1992;69:320-1.

28 Brimacombe J. The advantage of the LMA over the tracheal tube or facemask: a meta-analysis. Can $\mathcal{F}$ Anaesth 1995;42: tube or facen.

29 Yardy N, Hancox D, Strang T. A comparison of two airway aids for emergency use by unskilled personnel. Anaesthesia 1999;54:172-97. 
30 Baskett P, Nolan J, Parr M. Tidal volumes which are perceived to be adequate for resuscitation. Resuscitation 1996;31:231-4.

31 Weiler N, Latorre F, Eberle B, et al. Respiratory mechanics, gastric insufflation pressure, and air leakage of the laryngeal mask airway. Anesth Analg 1997;84:1025-8.

32 Dörges V, Ocker H, Hagelberg S, et al. Smaller tidal volumes with room-air are not sufficient to ensure adequate oxygenation during bag-valve-mask ventilation. Resuscitation 2000:44:37-41

33 Dörges V, Ocker H, Hagelberg S, et al. Optimisation of tidal volumes given with self-inflatable bags without additiona oxygen during simulated basic life support. Resuscitation 2000;43:195-9.
34 Wenzel V, Keller C, Idris AH, et al. Effects of smaller tidal volumes during basic life support ventilation in patients . Good ventilation, less risk? Resu-

tation 1999;43:25-9.
35 Guidelines 2000 for Cardiopulmonary Resuscitation and Emergency Cardiovascular Care-An International Consensus on Science Adult basic life support. Resuscitation 2000;46:29-71.

36 Guidelines 2000 for Cardiopulmonary Resuscitation and Emergency Cardiovascular Care-An International Consensus on Science Adult basic life support. Circulation 2000;102(suppl):I22-59.

37 Wenzel V, Idris AH. The current status of ventilation strategies during cardiopulmonary resuscitation (CPR). Curr Opin Crit Care 1997;3:206-13. 